\title{
Blockchain for Reorganization of Electronic Business Entrepreneurship
}

\author{
Dr. Panagiotis Gatomatis \\ University of Patras, Department of Management Science and Technology \\ School of Economics and Business \\ 1 MegalouAlexandrou Street \\ GR26334, Patras \\ Greece \\ Dr. Nikos Bogonikolos \\ CIC Rotterdam, Stationsplein 45 \\ 4th Floor, 3013 AK Rotterdam \\ Netherlands
}

\begin{abstract}
Blockchain technology is evolving to a dynamic element that could transform entire industries. Blockchain actually provides independent verification of data without a central, controlling party, a clearly decentralized way. This quality offers businesses both a higher level of precision and an increased ability to see the big picture in their operations. By storing and independently validating vast records of activities and transactions, companies are able to maintain a more accurate chain of custody and eventually manage an increasing number of assets.

Blockchain technology is envisioned to impact all major areas of businesses in the coming years, ultimately causing large-scale disruption to the global economy. At the same time, the basic concept of decentralized operations opens up a great number of benefits for business organizations.
\end{abstract}

Keywords: Blockchain, business, information, transparency, traceability, security, encryption, marketing

\section{Introduction}

Nowadays, more and more business experts believe that the blockchain technology will have profound effects on the nature of companies: how they are funded and managed, how they create value, and how they perform basic functions such as marketing, accounting, and incentivizing people. In some cases, software is expected to eliminate the need for many management functions [1].

The bright new capability for sellers and buyers to store and exchange value via some secure platform, ledger, or database without the need for traditional intermediaries: this is what blockchain technology is offering to businesses.

Stored and exchanged values are not saved in local repositories; they are represented by transactions recorded in a global spreadsheets or ledgers, and this leverages the resources of a large peer-to-peer network to verify and approve transactions.

A blockchain has several advantages. Most of them are widely known nowadays. We concentrate on three of them which we think as more relevant to the hereby discussed case. Initially, it is distributed: It runs on remote servers around the world, so there is no central database to hack. Further, it is public: Anyone can view it at any time because it resides on the network. And third, it is encrypted: It uses heavy-duty encryption to maintain security.

\section{The methodology of the blockchain technology}

Very briefly we can say the following: A distributed database, also known as a ledger, it is shared over a larger computing network. Copies of the shared ledgers exist in each one of the remote servers on the network.

Every block on the chain is a piece of data, which has been mathematically encrypted. Not only that, but there are numerous protocols required before each block is validated. This needs consensus from several others before it is added to the existing chain.

Because of the complex and highly encrypted design, the blockchain has no single point of failure. Hackers can't simply crack one server node to steal data. They would need to attack every node on the chain, simultaneously. 


\section{That means that blockchain is effectively un-hackable.}

Let's describe it alternatively: Blockchain transactions are continuously verified, cleared, and stored by the network in digital blocks that are connected to preceding blocks, thereby creating a chain. Each block must refer to the preceding block to be valid. This structure permanently time-stamps and stores exchanges of value, preventing anyone from altering the ledger. To steal anything of value, a thief would have to rewrite its entire history on the blockchain. Collective self-interest ensures the blockchain's safety and reliability. Therefore, blockchain provides a powerful mechanism for blowing traditional and centralized models, such as that of a commercial corporation, to bits in a safe and secure way.

And so, that is why we aforesaid that this technology paves the way for "an internet of value" [2], where buyers and sellers can store and exchange valuable assets directly, without any need for intermediaries.

\section{The induced change in the business framework}

Studies are alluding to the possibility that blockchain will be proven as revolutionary on a global scale quite soon. In a recent World Economic Forum it was estimated that a significant portion of global GDP will eventually be stored on blockchain. They predict $10 \%$ by the year 2027 [3]. This is quite big news. And then an equally big question arises: "Just how much will blockchain change the global economy?" The answer is "Too much!".

Blockchain technology is envisioned to impact all major areas of businesses in the coming years, ultimately causing large-scale disruption to the global economy. Actually, such a disruption has already begun. We will see in the below text six different ways that blockchain is changing business:

Financial management: The principles of blockchain technology which were developed to stop banking fraud can also be applied to financial management. Companies can use a blockchain-enabled system to track and manage their budget spending, all the way to final transaction. This reduces the risk of being overcharged for something small hidden in the chain of numerous transactions and also guarantees better performance in the long run. Thus, by adopting blockchain technology, financial executives can avail of better protection, ensuring their ROI doesn't suffer at the end of the day.

Human Resources management: Blockchain technology will soon modernize the hiring process, making it possible for human resources staff to verify candidates and current staff much faster. Any concerns of $3^{\text {rd }}$ parties interfering with inaccurate data on job applicants won't be further an issue. Furthermore, payroll will be streamlined, even for multinational corporations and companies with foreign staff. This is also expected to change how people save for retirement, as more people will invest progressively in blockchain-based crypto currencies in the future, rather than linking everything to their home country's currency and banks.

Business accounting: Adopting blockchain technology makes an accountant's life easier. With blockchain fraud is minimized. Control is maximized. Obscure data is minimized. Transparent data is maximized. Blockchain technology offers together total confidentiality and total anonymity. Transactions are recorded in a non-editable way via blockchain technology. This is revolutionary to accounting books keeping, maintaining and, of course, checking.

IT and Cybersecurity: Cybersecurity is by all means an incredibly vital part of current economy, globally, because in its absence the economy can be easily disrupted, hacked, and, in some sense, destroyed. Blockchain can shore up this valuable part of the economy. It can record digital transactions and interactions in a secure and efficient way, offering transparency and protection to form a solid foundation that companies can rely on.Blockchain can help businesses better strengthen their security. They can not only do transactions but also store data in a way to be assured on how the transactions are being made and also make their data immutable in nature.

General management: Blockchain transforms how businesses are organized and managed. This is a fact easily understandable. Blockchain allows companies to eliminate transaction costs and use resources on the outside as easily as resources on the inside. Vertical integration may continue to make sense in some situations (for manufacturing controlled pharmaceuticals, for example, or where companies have industry-leading strengths throughout the supply chain). But in most cases, networks based on blockchain will be better suited for creating products and services and for delivering value to stakeholders.

Sales Marketing: Just as a blockchain provides a way to obtain information about potential contractors and partners, it will be able to tell you about people or businesses who are potential customers. 


\section{The benefits of Blockchain technology to business sector}

Blockchain is a peer-to-peer network with no centralized authority. The core idea of decentralized concept opens up a great number of benefits for business organizations [4]. At the core, it provides the following benefits to them:

Distributed: Blockchain enables business organizations to benefit immensely from the decentralization. By using blockchain, business organizations can run their processes in distributed format. The needed computers (nodes) can be either owned by them or volunteer as there is no centralized database required in a distributed approach. Each peer has its own ledger, and this ensures that malicious actors cannot change the data.

Public: Business organizations are always keen to connect with their users. This means that they always have to have a particular process open to the end-user so that they can provide feedback or check information whenever needed. Blockchain supports business organizations in doing just that: create a federated blockchain network, as a mix of the public and private networks. The business organization will keep its critical processes and data in their private network while making proper use of the public features of the network. Thus, any user can check the information on the network anytime. This builds end-users trust and also makes business organizations accountable for their actions and deeds.

Security: Business organizations are always critical when it comes to the security of their trade secrets. They do not want their trade secrets to be a leak. Blockchain strengthens the security of their process and data. Blockchain uses high-level encryption to make sure that the data remains secure all the time. Many business organizations also implement a complete private encrypted blockchain network to make sure that their data never gets accessed by a person who is not authorized to do so.

Transaction Cost: Blockchain being independent on a centralized entity is cost-effective. The cost associated with processing a transaction is always lower compared to a centralized approach. Also, as the data is not stored in a centralized database, anyone within the business organization has the ability to access the data anywhere they want without the need to jump between conversations requesting the data. In some cases, the transaction cost can be completely removed - ensuring proper growth and efficiency.

No Intermediaries: There is a clear benefit of intermediaries. Business organizations now don't have to worry about $3^{\text {rd }}$ party solutions which act as intermediaries to provide service. This does off-load a lot of work from business organizations, but also bring the unnecessary risk associated with them. For instance, the whole work process can be halted because of the failure in the intermediate servers. If there is no backup, the business organization can lose a lot of value.

\section{The new concept of Blockchain-enabled e-commerce}

In the light of the above, it becomes understandable that block chain impacts well enough e-commerce. Actually it is the second time since 1982 when internet was born, that commerce faces such a revolutionary period. Back in 1982, the military ARPAnet was transformed into TCP/IP(Transmission Control Protocol and Internet Protocol) and after a little while pc-users could access email services and share documents over networks and the first browsers appeared when hypertext-based information was proposed by "World Wide Web". In 1994, the first services for processing online credit card sales appeared and one year later Verisign entered the market focusing on encryption and security of ecommerce servers.[5]

Nowadays, an equally important revolution is being expanding globally changing for good the conditions of ecommerce. Nowadays, the relationship between logistics performance and customer loyalty is much closer: the logistics service is directly provided to the customer, who has developed high expectations on the service level. The globalization of companies increased the complexity of the logistics model. It is now a key element to improve and integrate the information systems. New technologies are offering interesting ways of working around this challenge. Customer demands must be shared effectively; product and service deliveries must be tracked in real time provide visibility. End-to-end integration of product data is the main requirement for the supply chain industry. There have been $3^{\text {rd }}$ parties operating to establish process and data integration, by providing interoperability through the mapping and integration of organizations and systems.

So, blockchain technology is actually the next revolution to electronic data exchange over the internet between business partners. Blockchain technology promises to minimize the unnecessary use of $3^{\text {rd }}$ partyintermediaries. In this way, it would simplify B2B integration and enable micro level IoTintegration. 
The main challenges in e-commerce that blockchain technology can help to face are:

- Promoting transparency and trusted data,

- Reducing the blind spots in goods shipment,

- Accessing the suppliers' inventory: visibility on workflow between firms,

- Synchronizing demand planning and forecasting along the supply chain(s)

- Offering real-time data availability

- Improving traceability and compliance to standards

- Supporting Invoice Management

\section{Conclusions}

It is quite clear that the revolution that is being induced in the operational conditions of the business organizationsand e-commerce has quite a size and impact. Not only in the operational conditions but also in the decision-making process. And not only in the operational conditions and the decision-making process but also in the methodology and the security of the financial transactions. If we keep counting the affected sectors of the everyday operations of the business organizations, we will figure out that such a list is multitudinous. It becomes obvious that blockchain technology has the potential to significantly change a wide spectrum of business and e-commerce processes. As this technology is gaining traction and making its presence known in business processes, it will perhaps be role of process experts to ensure that the value created comes from exploring the opportunities this technology can enable in business processes rather than just replacing existing technologies with a new one. The overall feeling is that blockchain is changing everything in the electronic business entrepreneurship sector. It has great potential to introduce new and improved methodologies and ways towards a more secure, trusted, transparent and efficient business ecosystem.

\section{References}

Hileman, Garrick, (2016) [Online] Available: http://www.coindesk.com/state-of-blockchain-q1-2016/ (June 15, 2016)

Tapscott, Alex \& Don, (2016) [Online] Available: https://sloanreview.mit.edu/article/how-blockchain-will-changeorganizations/?utm_source=author\&utm_medium=referral\&utm_campaign=smr-marketing $($ December 7 , 2016)

Korizky, Roman, (2015) [Online] Available: http://www.coinfox.info/news/3184-world-economic-forum-survey-10-ofglobal-gdp-may-be-stored-with-blockchain-technology-by-2027 (September 26, 2015)

Logaras, Konstantinos, (2018) [Online] Available: https://www.lawspot.gr/nomika-nea/h-tehnologia-blockchain-oiefarmoges-kai-oi-nomikes-ptyhes-tis (June 14, 2018)

Roos, Dave. (2008) [Online]http://money.howstuffworks.com/history-e-commerce1.htm 